their eggs in Great Britain and Northern Ireland from now until the end of 1942 (the following species excepted-house sparrow, starling and wood-pigeon) should be prohibited during 1941 and 1942, and the results of such action carefully reviewed and any further action considered at the end of 1942 .

The Yorkshire Museum,

York. Jan. 21.

1 Proc. Berwickshire Nat. Club, 9, 304-572 (1881).

Trans. Norf. and Norw. Nat. Soc., 5, 191-195.

3 Brit. Birds, 11, 266-271, and ibid., 12, 26-35 (1918).

4 Brit. Birds, 34, 118-132, and ibid., 142-155 (1940).

5 National Review, 234-239 (1918).

\section{Conical Refraction in Naphthalene Crystals}

THE birefringence of many organic crystals of the aromatic class is large, and when the intermediate index differs widely from the upper and lower indexes, the angles of internal and external conical refraction assume very high values. These angles in naphthalene, for example, are both about $13^{\circ} 45^{\prime}$, which may be compared with $1^{\circ} 54^{\prime}$ and $1^{\circ} 44^{\prime}$ respectively in the classical case of aragonite. By fusion followed by very slow solidification, it is fairly easy to obtain transparent blocks of naphthalene and other aromatic compounds. When suitably cut and mounted between glass cover-slips, naphthalene crystals exhibit the phenomena of conical refraction in a very striking way, and enable their features to be critically examined.

The photographs reproduced with this note are the images of a fine illuminated pinhole held against the face of a naphthalene crystal $2 \mathrm{~mm}$. thick and viewed through it in the appropriate direction. In the first of the series, the pinhole is seen in perfect focus and appears as a very sharp and perfect circle. The three other pictures illustrate the effect of moving the focus away from the pinhole, until, finally, in the last picture, the second surface of the crystal is
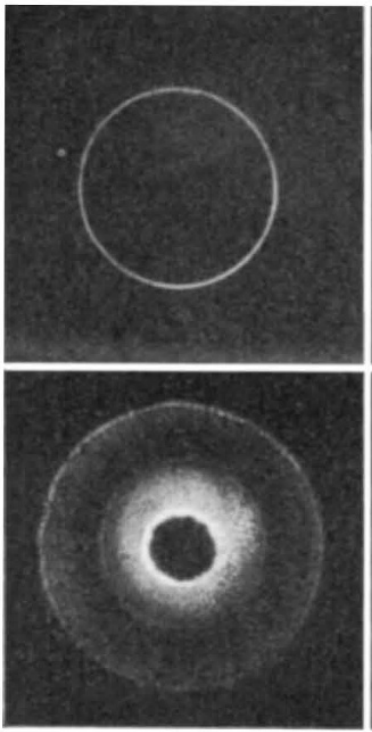

3
2
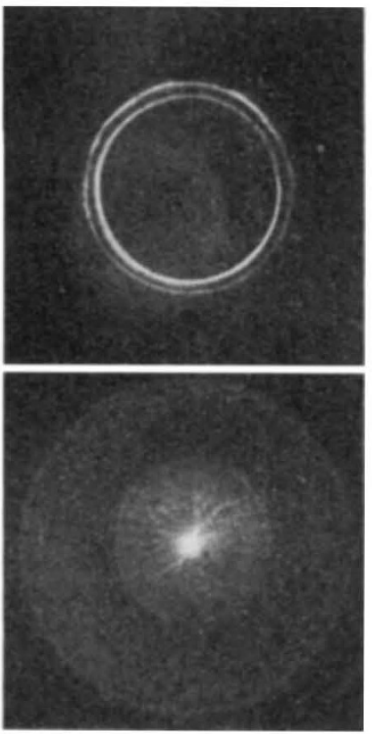

4
Conical Refraction in Napthalene Crystals. in focus. The central bright spot seen in the fourth picture is actually an image ${ }^{x}$ of the fine pinhole used, and appears at the point where the axis of single-ray velocity meets the rear surface. This bright spot continues to be the most conspicuous feature in the field for a great distance behind the crystal.

It will be seen on a comparison of the first and second pictures that, at least in the case of naphthalene, the so-called Poggendorf dark circle vanishes when the pinhole is seen in perfect focus.

C. V. RAMAN.

V.S. RAJAGOPALAN.

Department of Physics,

T. M. K. Nedungadi.

Indian Institute of Science,

Bangalore.

Dec. 19

${ }^{1}$ Raman, C. V., Nature, 107, 747 (1921), and Phil. Mag., 43, 510 (1922).

\section{Temperature Dependence of Adiabatic Compressibility}

IT is well known that the adiabatic compressibility $\beta_{\varphi}$ of a liquid increases fairly rapidly with rise in temperature. The rise becomes more rapid as the temperature increases. With many liquids the adiabatic compressibility increases by about 60 per cent for a rise of temperature from $0^{\circ}$ to $60^{\circ}$, or $\frac{1}{\sqrt{\beta_{\varphi}}}$

decreases by 21 per cent. A large part of the high initial compressibility of ordinary liquids is connected with the nearness of the critical point liquid gas, for compressibility in the gas phase is high and at the critical point itself compressibility is infinite ${ }^{1}$. Hence the absolute value of the compressibility depends on how far the temperature is from the critical point.

The characteristic frequency of vibration of molecules in a liquid is given by

$$
\nu=S\left(\frac{3 N}{4 \pi V}\right)^{1 / 3},
$$

where $S$ is the velocity of sound in the liquid and $N$ and $V$ are Avogadro's number and molar volume respectively ${ }^{2}$;

$$
\text { or } \nu=c \sqrt{\frac{V^{1 / 3}}{M \beta_{\varphi}}},
$$

for a given liquid, where $c$ is a constant and $M$ the molecular weight.

In the light of the available data ${ }^{3}$ on the compressibility of a number of liquids, the above expression has been examined in relation to its temperature variation, and it is found that the expression decreases linearly with temperature according to the relation

$$
\sqrt{\frac{\overline{V^{1 / 3}}}{M \beta_{\varphi}}}=k\left(\theta_{c}-\theta\right),
$$

where $\theta_{c}$ is the critical temperature, $\theta$ is any temperature and $k$ is a constant characteristic of the

\begin{tabular}{|c|c|c|c|}
\hline \multicolumn{3}{|l|}{ Liquid } & $k$ \\
\hline Benzene .. & . & . & $110 \cdot 8$ \\
\hline Carbon tetrach & loride & .. & $77 \cdot 5$ \\
\hline Ether .. & .. & .. & $121 \cdot 8$ \\
\hline Chlorobenzene & .. & .. & $80 \cdot 8$ \\
\hline Toluene .. & .. & .. & $92 \cdot 6$ \\
\hline Acetic acid & .. & .. & $93 \cdot 6$ \\
\hline
\end{tabular}
liquid. In the following table are given values of $k$ for a number of liquids : 